\title{
PERCEPCIÓN DE LOS ESTUDIANTES UNIVERSITARIOS RESPECTO A TÉCNICAS UTILIZADAS POR SUS DOCENTES EN EL AULA
}

\section{PERCEPTION OF COLLEGE STUDENTS REGARDING YOUR TECHNIQUES FOR TEACHERS IN THE CLASSROOM}

\author{
Ricardo R. Vega Bois ${ }^{1}$
}

\section{RESUMEN}

El desempeño de los docentes en el aula, principalmente sus metodologías e instrumentos utilizados tienen más allá de la simple entrega de elementos que son parte del proceso enseñanza - aprendizaje, impactos que inciden en aspectos fundamentales para la obtención y cumplimiento de metas, tales como la generación de interés, motivación real y efectiva, percepción de utilidad y comprensión adecuada de contenidos por parte del estudiante.

La investigación se genera por una parte por la inquietud de conocer la percepción que tienen los estudiantes de auditoría respecto a las técnicas utilizadas en el aula por sus docentes y por otra, con la recabar antecedentes que permitan mejorar el desempeño de los docentes universitarios en el aula. El estudio estuvo acotado a estudiantes de la carrera de Auditoría de la Universidad de Los Lagos, con resultados que pueden generalizarse en la medida que es tos se contextualicen adecuadamente.

La investigación, documental y de campo, con altos componentes cualitativos se inició con la recolección de opiniones en base a una encuesta técnicamente diseñada y validada, convergiendo a un segundo proceso de análisis, parametrización y conclusiones, respondiendo así las inquietudes planteadas, generando paralelamente algunas recomendaciones de carácter general.

Los principales resultados establecieron que por sobre lo que generalmente se puede pensar, los estudiantes valoran aspectos técnicos y de adecuada exigencia por sobre situaciones y/o acciones facilitadoras del avance curricular sin mayor grado de dificultad. A la vez se descarta que los medios técnicos de apoyo por sí solos sean suficientes para cumplir un adecuado proceso de enseñanza aprendizaje.

Palabras claves: Docentes, Estudiantes, Técnicas Utilizadas.

Recepción: 10/06/2016. Aprobación: 14/09/2016.

\begin{abstract}
ABSTRAC
The performance of teachers in the classroom, mainly methodologies and tools used are beyond the simple delivery of items that are part of the teaching - learning process, impacts that affect fundamental aspects for obtaining and achieving goals, such as generating interest, real and effective motivation, perceived usefulness and adequate understanding of content by the student.
\end{abstract}

1 Académico del Departamento de Ciencias Administrativas y Económicas de la Universidad de Los Lagos, Osorno, Chile. e-mail: ricardo.vega@ulagos.cl 
The research is generated on the one hand the concern to know the perception students audit regarding the techniques used in the classroom by their teachers and on the other, to obtain background to improve the performance of university teachers in the classroom. The study was limited to students studying Audit at the University of Los Lagos, with results that can be generalized to the extent that it is properly contextualize cough.

Research, documentary and field, with high qualitative components began with the collection of opinions based on a technically designed and validated survey converging to a second round of analysis, parameterization and conclusions, thus answering the concerns raised, generating parallel some general recommendations.

The main results established that what usually be thinking, students value adequate technical and demanding aspects over situations and / or enabling actions of curriculum progress without much difficulty. At the same time it ruled out that the technical means to support themselves are sufficient to meet appropriate teaching - learning

Keywords: Teachers - Students -Techniques Used.

\section{INTRODUCCIÓN}

La motivación en el aula de los estudiantes es muchas veces gravitante en el rendimiento que puedan estos lograr, debiendo ser el docente mediador entre la accesibilidad a los aprendizajes y la adquisición de competencias, debiendo necesariamente buscar y utilizar apropiadamente diversas técnicas y herramientas para cumplir a cabalidad su cometido.

Independiente del nivel de motivación de entrada del estudiante, esta irá variando, para mejor o peor, siendo muchos los factores que afectan la motivación de un estudiantes (Bligh, 1971; Sass, 1989), como por ejemplo el interés en la materia, la percepción de su utilidad, la paciencia del alumno y las técnicas que utilice el docente en el aula, entre otras.

Con la finalidad de capturar estas percepciones se consideraron dimensiones específicas como cualidades del docente, uso de material de apoyo, organización de contenidos, nivel de dificultad, participación activa del estudiante, uso de tecnología en el aula, conexión profesor - estudiante, uso de ejemplos, además de referencias y técnicas de evaluación.

La investigación si bien fue acotada a un contexto en particular, permite evidenciar percepciones de estudiantes del área de Auditoría de la Universidad de Los Lagos respecto al uso y técnicas en el aula y la opinión que tienen los estudiantes al respecto, considerando dimensiones transversales que permiten en su contexto y análisis detallado concluir e identificar importantes indicadores, destacando la importancia que tienen la motivación del estudiante en el aula (García, 2005).

Es importante considerar que las estrategias de aprendizaje ponen de manifiesto la implicación en la enseñanza de los diferentes tipos de pensamiento y estrategias metacognitivas (Genovard y Gotzens, 1990). El poseer conciencia de las propias estrategias metacognitivas inducen a su aplicación en situaciones de aprendizaje, resolución de problemas y memorización (Melot, 1990), existiendo intentos de establecer relaciones entre las estrategias de aprendizaje y el rendimiento académico (Cano y Justicia. 1998).

La aproximación de los estilos de enseñanza al estilo de aprendizaje requiere como señala Bernard (1993) que los docentes comprendan la gramática mental de sus estudiantes derivada de los conocimientos previos y del conjunto de estrategias, guiones o planes utilizados en el aula.

Respecto a estudios similares encontramos entre otros a P Bourdieu, JC Passeron (2009) en Los herederos: Los estudiantes y la cultura. (Consistió en explicar los mecanismos de elección de elegidos y de producción de las percepciones sociales de los estudiantes durante algún tiempo), citado por 538 publicaciones. (http: / / atlas.umss. edu.bo:8080/xmlui/handle/123456789/877). A Polo, JM Hernández, C Pozo (1996) en Evaluación del estrés académico en estudiantes universitarios, Citado en 138 publicaciones. También en Scielo. Org. Full View a Fedman, L Goncalves, G Chacon-Puignau (2008), con Relaciones entre estrés académico y rendimiento en estudiantes, Universitas - scielo.org.co., también a FD Betoret, PJ Jiménez, JR Remírez - Psicothema (2004) en Percepción del proceso de enseñanza - aprendizaje y su incidencia en el rendimiento - unioviedo.net (aconsejan introducir otras variables mediadoras entre la percepción y el aprendizaje), citado 17 veces. En Scileo.org.pe a RM Méndez García (2009). Los estudiantes: su percepción sobre lo que 
implica "ser estudiantes" $y$ "hacer universidad". Revista de Investigación Educativa, 2009 - revistas. um.es, citado 9 veces en publicaciones a la fecha.

\section{METODOLOGÍA, MATERIAL Y MÉTODOS}

La investigación se circunscribe a la Carrera de Contador Público y Auditor de la Universidad de Los Lagos. El primer proceso se fundamenta en realizar una descripción del problema objeto de investigación a base de elementos teóricos y prácticos. Para cumplir con la tarea anterior, se utilizaron los procedimientos regulares de investigación documental y de campo, de carácter descriptivo, en base a la aplicación del método científico, esta primera parte de la investigación tiene características esencialmente cuantitativas.

En el segundo proceso en función de los resultados obtenidos de la investigación descriptiva, basándose de los principios de la investigación cualitativa, se optó por generar análisis y propuestas respecto a las técnicas utilizadas en aula por los docentes de la carrera. Fue de tipo exploratoria, descriptiva y propositiva, puesto que los resultados del estudio nos darán argumentos para el planeamiento y planteamiento de mejoras en las técnicas que los docentes utilizamos en el aula.

Para obtener la información requerida se elaboró una encuesta en base a una metodología de identificación de dimensiones, la que fue aplicada a estudiantes de 2 do año y superiores, excluyéndose a los de primer año principalmente por su corta estadía en la universidad y carrera.

En base a la inquietud de conocer la percepción que tienen los estudiantes de auditoría respecto a las técnicas utilizadas en el aula por sus docentes y a recabar antecedentes que permitan aportar al mejoramiento del desempeño de los docentes universitarios en el aula, se plantearon los siguientes objetivos.

\subsection{Objetivo general}

Analizar la percepción que tienen los estudiantes de segundo año y superiores de auditoría, al primer semestre de 2015, respecto a las técnicas utilizadas por sus docentes en el aula.

\subsection{Objetivos especificos}

- Caracterizar a los estudiantes de la carrera de Contador Público y Auditor en estudio, mediante la revisión de bases de datos institucionales, aplicando criterios de inclusión y exclusión para la determinación de la muestra.

- Delimitar a través de una determinación técnica basada en estudios previos relacionados, dimensiones del quehacer docente en el aula.

- Detectar, mediante el análisis de la información recabada, las percepciones que tienen los estudiantes respecto a las técnicas utilizadas por sus docentes en el aula

- Delinear en base a la parametrización de la información obtenida, los diferentes grados de valorización que le otorgan los estudiantes de Auditoría a las dimensiones de técnicas utilizadas en el aula en estudio.

\subsection{Variables}

En base a la aplicación del instrumento de captura de percepción, el cual dirige el estudio hacia estudiantes considerados en la muestra, los cuales se convierten en informantes claves, se determinaron las siguientes variables:

- Relación entre los docentes y los estudiantes de Auditoría (Percepción de los estudiantes, variable dependiente)

- Relación entre docentes y jefe de carrera con respecto a la comunicación que estos tienen, análisis de metodologías y de resultados obtenidos (Variable independiente)

- Experiencia de los docentes frente a las herramientas y procedimientos de mejora que entrega la universidad (Variable independiente).

\section{4. Área de estudio, población y muestra}

El estudio se aplicó a toda la Carrera de Contador Público y Auditor de la Universidad de Los Lagos, estudiantes vigentes a la fecha de su aplicación.

La población total de estudiantes vigentes a la fecha del estudio fue de 103 alumnos en todos los niveles. Buscando representatividad real de estudiantes que hubiesen participado durante ya un tiempo en el proceso formativo, teniendo estos ya una opinión formada respecto de las dimensiones de percepción indagadas, se decidió no considerar a los estudiantes de primer año en la muestra, direccionando el universo a los estudiantes de segundo a quinto año, lo que acotó la población objetivo a 85 estudiantes.

La muestra válida arrojó un $80 \%$ del univer- 
so con un grado de confianza de $95 \%$, con un margen de error del $5 \%$. Por ende se trabajó con una muestra de 70 estudiantes ( 20 de 2 do año, 18 de 3ero, 17 de 4 to y 15 de 5 to año).

Criterios de inclusión: Estudiantes de la Carrera Contador Púbico y Auditor vigentes a la fecha del estudio.

Criterios de exclusión: Estudiantes de la Carrera Contador Púbico y Auditor de primer año.

\subsection{Técnica e Instrumento}

Se preparó un instrumento de medición (encuesta) en la cual se ponderó de 1 a 7 el valor que para cada ítem con sus respectivas múltiples dimensiones, le otorga el estudiante.

Para definir las dimensiones a abordar se utilizó la siguiente matriz, basada en La Matriz de Percepciones de Carlos Alberto Sierra Serna (http: / / es.slideshare.net/ carlosierra/matrizde-percepciones-5076043, consultada en abril de 2015):

Tabla I: Establecimiento de dimensiones.

\begin{tabular}{|c|c|c|}
\hline ASPECTOS DEL DOCENTE & TIPO DE DIMENSIÓN & DIMENSIÓN \\
\hline Actitudinales & Cualitativa & $\begin{array}{l}\text { - Cualidades del Docente* } \\
\text { - Organización } \\
\text { - Incentivo a la participación } \\
\text { - Conexión profesor - estudiante* }\end{array}$ \\
\hline Técnicos/Profesionales & Mixta & $\begin{array}{l}\text { - Uso de material de apoyo } \\
\text { - Organización de contenidos } \\
\text { - Nivel de dificultad } \\
\text { - Uso de tecnología } \\
\text { - Uso de ejemplos } \\
\text { - Técnicas de evaluación } \\
\text { - Cualidades del docente* } \\
\text { - Conexión profesor - estudiante* }\end{array}$ \\
\hline
\end{tabular}

*Se considera cualidades del docente y la conexión docente - estudiante en dos dimensiones pues se produce una combinación de la actitudinal y de ascendencia profesional cuando su manifestación es adecuada.

Fuente: Elaboración propia 2016 a partir de Matriz de Percepciones (Sierra Serna, 2015). 
Para la definición de las preguntas que cubrieran dichas dimensiones se utilizó la siguiente matriz, basada en la anterior:

Tabla II: Interrogantes a considerar.

\begin{tabular}{|c|c|c|c|}
\hline ASPECTO & $\begin{array}{l}\text { OBJETIVO DE } \\
\text { EVALUACIÓN }\end{array}$ & INTERROGANTES & EXCLUSIONES* \\
\hline $\begin{array}{l}\text { Cualidades del } \\
\text { docente }\end{array}$ & $\begin{array}{l}\text { Dimensionar la percepción } \\
\text { que tienen los estudiantes res- } \\
\text { pecto a ciertas cualidades que } \\
\text { poseen o carecen sus docentes }\end{array}$ & $\begin{array}{l}\text { Respecto a dominio, cumpli- } \\
\text { miento de fechas, lenguaje, } \\
\text { experiencia, actitud, indepen- } \\
\text { dencia de material de apoyo }\end{array}$ & $\begin{array}{l}\text { Edad, sexo, antigüedad, } \\
\text { nacionalidad, etnia, proce- } \\
\text { dencia. }\end{array}$ \\
\hline Organización & $\begin{array}{l}\text { Dimensionar la percepción } \\
\text { que tienen los estudiantes } \\
\text { respecto a la incidencia de una } \\
\text { adecuada organización de las } \\
\text { cátedras. }\end{array}$ & $\begin{array}{l}\text { Si existe conocimiento previo, } \\
\text { importancia de esta, cumpli- } \\
\text { miento de planificaciones y } \\
\text { recuperación efectiva de clases }\end{array}$ & $\begin{array}{l}\text { Entrega/publicación de } \\
\text { programas, reglamento de } \\
\text { carrera, actas oficiales de } \\
\text { asignaturas. }\end{array}$ \\
\hline $\begin{array}{l}\text { Material de } \\
\text { apoyo }\end{array}$ & $\begin{array}{l}\text { Obtener la percepción que } \\
\text { tienen los estudiantes respecto } \\
\text { al material de apoyo entregado } \\
\text { por los docentes y su utiliza- } \\
\text { ción en el aula. }\end{array}$ & $\begin{array}{l}\text { Valorización de este, desa- } \\
\text { rrollo de casos, metodología } \\
\text { aplicada en el aula. }\end{array}$ & \\
\hline $\begin{array}{l}\text { Nivel de difi- } \\
\text { cultad }\end{array}$ & $\begin{array}{l}\text { Dimensionar la percepción } \\
\text { respecto a la valorización del } \\
\text { estudiante del grado de pro- } \\
\text { fundidad de las asignaturas. }\end{array}$ & $\begin{array}{l}\text { Valorización del grado de } \\
\text { profundidad de las asignatu- } \\
\text { ras, relación dificultad/entrega } \\
\text { del docente. }\end{array}$ & $\begin{array}{l}\text { Asignaturas generales y/o } \\
\text { de formación complemen- } \\
\text { taria. }\end{array}$ \\
\hline $\begin{array}{l}\text { Participación } \\
\text { activa }\end{array}$ & $\begin{array}{l}\text { Percibir la valorización que } \\
\text { los estudiantes le otorgan a su } \\
\text { participación en el aula. }\end{array}$ & $\begin{array}{l}\text { Interacción en el aula, asun- } \\
\text { ción de roles, fomento a la } \\
\text { participación }\end{array}$ & $\begin{array}{l}\text { Actividades de participa- } \\
\text { ción obligatoria. }\end{array}$ \\
\hline Uso de tecnología & $\begin{array}{l}\text { Percibir el grado de importan- } \\
\text { cia que el estudiante le otorga } \\
\text { al uso de tecnología en el aula. }\end{array}$ & $\begin{array}{l}\text { Importancia otorgada, valo- } \\
\text { rización e técnicas utilizadas, } \\
\text { prescindencia }\end{array}$ & $\begin{array}{l}\text { Asignaturas que requieren } \\
\text { obligatoriamente el uso de } \\
\text { laboratorios de computa- } \\
\text { ción o similares. }\end{array}$ \\
\hline $\begin{array}{l}\text { Conexión docen- } \\
\text { te/estudiante }\end{array}$ & $\begin{array}{l}\text { Dimensionar la valorización } \\
\text { que le otorgan los estudiantes } \\
\text { a su relación directa con los } \\
\text { docentes. }\end{array}$ & $\begin{array}{l}\text { Relación con aprendizajes, } \\
\text { valorización de la receptibili- } \\
\text { dad de los docentes, referencia } \\
\text { profesional. }\end{array}$ & \\
\hline $\begin{array}{l}\text { Ejemplos - } \\
\text { referencias }\end{array}$ & $\begin{array}{l}\text { Percibir la valorización que } \\
\text { le otorgan los estudiantes al } \\
\text { uso de ejemplos y referencias } \\
\text { apropiadas y válidas por parte } \\
\text { de sus docentes. }\end{array}$ & $\begin{array}{l}\text { Valorización de ejemplos } \\
\text { aplicados, de referencia a } \\
\text { situaciones profesionales y de } \\
\text { análisis de contingencia. }\end{array}$ & \\
\hline $\begin{array}{l}\text { Técnicas de } \\
\text { evaluación }\end{array}$ & $\begin{array}{l}\text { Percibir preferencias respecto } \\
\text { a las evaluaciones por parte de } \\
\text { los estudiantes. }\end{array}$ & $\begin{array}{l}\text { Preferencias sobre el tipo de } \\
\text { evaluación, sobre las formas } \\
\text { de evaluación y la pondera- } \\
\text { ción que estas deben a juicio } \\
\text { del estudiante, tener. }\end{array}$ & $\begin{array}{l}\text { Asignaturas no discipli- } \\
\text { nares }\end{array}$ \\
\hline
\end{tabular}

*Las exclusiones son recomendadas para aislar factores y / o elementos de dispersión que alejen del cumplimiento de objetivos.

Fuente: Elaboración propia 2016 a partir de Matriz de Percepciones (Sierra Serna, 2015). 
Como técnica base del estudio por tanto, se utilizó una encuesta aplicada vía formulario tipo escala modificado de Likert (Ver anexo), confeccionando las preguntas en base a las definiciones estructuradas anteriormente.

\subsection{Validez y Confiabilidad del Instrumento}

El instrumento aplicado se validó desde dos ópticas: la primera se remitió para ello a experta en metodología quién efectuó un análisis de cumplimiento de objetivos y pertinencia de la encuesta aplicada. Para obtener una segunda validación, se comparó a modo de prueba de control con resultados obtenidos por el investigador con 20 estudiantes de la carrera de Ingeniería en Administración en otra universidad, aplicada en 2012, concluyéndose que los resultados obtenidos son comparables.

Lo anterior buscó un índice de fiabilidad cercano a 1,0. Con una variación comparativa entre el piloto y la muestra fue de $15 \%(0,15)$ y el error de $3 \%(0,03)$, otorgando por ende una fiabilidad de $83 \%(0,83)$. Como la variación de error disminuye en valor (o como los puntajes del cues- tionario son más consistentes) el coeficiente de fiabilidad es cercano al valor de 1.00. Esto indica un buen juego de datos. Es importante destacar que este índice es de fiabilidad del instrumento, distinto a de la muestra indicada anteriormente en el punto 2.6.

\subsection{Plan de recolección, Presentación, Análisis e Interpretación de Datos}

La recolección de datos se realizó en aulas en mayo de 2015, oportunidad en que se explicó la finalidad del instrumento y la importancia de su aplicación. La encuesta en cada oportunidad ocupó un tiempo aproximado de 20 minutos. Luego de la recolección de datos, estos fueron tabulados por dimensión y codificados a través de grupos y subgrupos, asignándole un igual a 1 a cada tendencia marcada.

\section{RESULTADOS}

La tabulación de los resultados nos entregó los siguientes indicadores: 
Tabla III: tabulación de resultados.

\begin{tabular}{|c|c|c|}
\hline DIMENSIÓN & VARIABLES DESTACADAS & \% DE MENCIÓN \\
\hline $\begin{array}{l}\text { Cualidades del } \\
\text { docente }\end{array}$ & $\begin{array}{l}\text { Grados adecuados de } \\
\text { independencia del material de } \\
\text { apoyo en el aula }\end{array}$ & $90 \%$ \\
\hline Organización & $\begin{array}{l}\text { Recuperación efectiva de clases } \\
\text { no realizadas }\end{array}$ & $85 \%$ \\
\hline Nivel de dificultad & $\begin{array}{l}\text { No preferencia de asignaturas } \\
\text { fáciles por sobre las más } \\
\text { exigentes }\end{array}$ & $74 \%$ \\
\hline Material de apoyo & $\begin{array}{l}\text { Valorización de metodología de } \\
\text { casos desarrollados en el aula } \\
\text { con apoyo docente y } \\
\text { bibliográfico }\end{array}$ & $100 \%$ \\
\hline Participación activa & $\begin{array}{l}\text { Clases interactivas, asunción de } \\
\text { rol activo, fomento a la } \\
\text { participación }\end{array}$ & $86 \%$ \\
\hline \multirow{2}{*}{$\begin{array}{l}\text { Uso de tecnología } \\
\text { en el aula }\end{array}$} & $\begin{array}{l}\text { Preferencia clase tradicional sin } \\
\text { uso de tecnología }\end{array}$ & $44 \%$ \\
\hline & $\begin{array}{l}\text { Preferencia por el uso de } \\
\text { tecnología en el aula }\end{array}$ & $56 \%$ \\
\hline $\begin{array}{l}\text { Conexión profesor - } \\
\text { estudiante }\end{array}$ & \begin{tabular}{|l|} 
Empatía de los docentes, \\
atención de consultas, docentes \\
referentes profesionales
\end{tabular} & $97 \%$ \\
\hline $\begin{array}{l}\text { Uso de ejemplos - } \\
\text { referencias }\end{array}$ & $\begin{array}{l}\text { Ejemplos apropiados, referencia } \\
\text { a situaciones profesionales } \\
\text { verídicas, análisis de } \\
\text { contingencia nacional. }\end{array}$ & $94 \%$ \\
\hline $\begin{array}{l}\text { Técnicas de } \\
\text { evaluación }\end{array}$ & $\begin{array}{l}\text { Talleres y evaluaciones grupales } \\
\text { con asesoría del docente. }\end{array}$ & $100 \%$ \\
\hline
\end{tabular}

Fuente: Elaboración propia a partir de tabulación de resultados obtenidos.

La interpretación de los resultados en cada ítem consultado se realizó considerando la concentración de tendencias, permitiendo tener una percepción a la fecha del estudio, lo que permitirá en investigaciones futuras evaluar posibles cambios y motivos de estos.

Aportes no inducidos (comentarios de los estudiantes):
1. Relacionados con las evaluaciones:

1.1.Sugieren eliminar Prueba Integradora (6 menciones)

1.2. Qué las notas de proceso tengan una ponderación mayor (2 menciones)

1.3. Más evaluaciones prácticas y en terreno (2 menciones)

1.4. Adecuar de acuerdo a las características de la carrera la Evaluación Final (Sic, 1 mención) 
2. Sobre metodologías y técnicas de los docentes:

2.1. Clases teóricas más didácticas favoreciendo el aprendizaje ( 2 menciones)

2.2. Readecuar las técnicas docentes hacia aplicaciones prácticas de la especialidad (1 mención)

2.3. Queja: "Los profesores de la rama de auditoría demuestran desinterés, falta de conocimientos e incapacidad de enseñar: insultante" (Sic, 1 mención).

3. Sobre capacidades docentes:

3.1. “No siempre un profesor con grandes títulos está capacitado para enseñar, a veces falta más empatía profesor - alumno" (Sic, 1 mención)

La interpretación, más que una operación distinta, es un aspecto especial del análisis y su objetivo es "buscar un significado más amplio a las respuestas mediante su trabazón con otros conocimientos disponibles" (Selltiz, 1970) que permitan la definición y clarificación de los conceptos y las relaciones entre éstos y los hechos materia de la investigación.

\section{CONCLUSIONES}

Interpretando y analizando las tendencias detectadas para cada uno de las dimensiones en estudio, podemos en forma general en algunos casos y con bastante certeza en otros, concluir primero en cada ámbito y posteriormente en forma general, respecto a los estudiantes de Auditoría de la Universidad de Los Lagos:

\section{1. Ámbito: Cualidades del docente}

Los estudiantes de Auditoría de la universidad de Los Lagos valoran en gran medida cualidades de sus docentes, como lo son la experiencia, el dominio profesional de su área de competencia, lenguaje y cumplimiento de horarios. Destaca en forma especial en este ítem, aparte del reconocimiento a la experiencia, la importancia alta otorgada al nivel de entusiasmo y la condición de alegres de sus docentes, convirtiéndose por tanto en cualidades complementarias de alta valorización por parte de los estudiantes.

\section{2. Ámbito: Organización}

La organización y planificación docente es en todas sus dimensiones valorada positivamente por los estudiantes de Auditoría de la Universidad de Los Lagos, teniendo por ende un carácter de importante para ellos. Destaca a la vez el sub ítem de "recuperación eficaz de clases no efectuadas" pasando a ser más que una obligación académica una condición necesaria para la gran mayoría de los encuestados.

\section{3. Ámbito: Material de apoyo}

Se manifiesta una muy buena valorización a la entrega, uso y desarrollo asistido de material complementario al desempeño en el aula. Se destaca en este ítem la alta consideración que se le otorga a la metodología de casos y su desarrollo en clases, siendo lo anterior muy práctico y útil en las asignaturas de formación profesional en el ámbito de la Auditoría.

\section{4. Ámbito: Nivel de dificultad}

Por sobre todo paradigma que se pueda tener al respecto, los estudiantes de Auditoría de la universidad de Los Lagos no prefieren asignaturas catalogadas de fáciles como puede pensarse generalmente en la academia, indicando a la vez que las difíciles y/o complejas no lo son tanto con buenos docentes. Lo anterior entrega una dimensión de análisis que nos llevarán a tener indicadores de medición de calidad y de fortalecimiento de la docencia importantes desde la óptica de los estudiantes.

\section{5. Ámbito: Participación activa}

Se valora el incentivo a participar en clases, pero a la vez se manifiestan algunos grados de timidez, siendo esta una característica que vemos generalmente en nuestros alumnos de primeros años de la carrera. Lo anterior permitirá establecer algunos indicadores de trabajo en aula para los docentes, evitando que se produzca el "efecto mateo".

\section{6. Ámbito: Uso de tecnología en el aula}

Hay una valorización mediana al uso de la tecnología en el aula, al parecer no se le considera gravitante en el proceso de enseñanza aprendizaje. Destaca en este ítem que un porcentaje no menor optan por clases "tradicionales" versus el uso de tecnología de apoyo, pero a la vez se considera medianamente importante el uso de esta, lo que es claramente un indicador que de utilizarse, este uso debe ser ponderado 
y apropiado para los objetivos de aprendizajes.

\section{7. Ámbito: Conexión profesor - estudiante}

Los estudiantes de Auditoría de la universidad de Los Lagos se inclinan por la existencia de una conexión activa y constante con sus docentes, destacándose la atención de consultas. A la vez es importante indicar el alto porcentaje de respuestas en las que se define a algunos docentes como buenos referentes profesionales.

\section{8. Ámbito: Ejemplos y referencias}

Se valora en gran medida el uso de ejemplos prácticos reales y a la vez, el análisis del docente de la contingencia nacional desde varios ámbitos. Destaca en este ítem la alta valorización que le otorgan los estudiantes de Auditoría de la Universidad de Los Lagos a las referencias de situaciones profesionales verídicas (experiencias).

\section{9. Ámbito: Técnicas de evaluación}

Lidera las apreciaciones de los estudiantes de Auditoría de la Universidad de Los Lagos en forma positiva, la aplicación de Talleres y/o Evaluaciones Grupales, con asesoría del docente, lo que es complementario a lo manifestado en el ítem 5.3. respecto a la metodología de casos como apoyo docente.

La labor docente en el aula es gravitante para el logro de objetivos y el éxito del proceso enseñanza - aprendizaje en esta disciplina profesional y el presente estudio nos entrega apreciaciones obtenidas desde los usuarios directos de nuestra labor, los estudiantes. A modo de conclusión general podemos indicar que los estudiantes en cada ámbito, reafirman muchas apreciaciones que podemos tener los docentes respecto a nuestra labor en la formación de futuros Auditores, especialmente respecto a la calidad de contenidos y a los diferentes niveles de dificultad que estos deben enfrentarse para adquirir así las competencias necesarias, no deseando un camino fácil, sino que allanado por sus docentes (adaptado de Tobón, 2006).

La experiencia y los conocimientos actualizados del docente son altamente valorados, lo que induce a potenciar la relación universidad - empresa que debe existir en nuestro quehacer profesional. A la vez esta entrega debe ser con niveles de motivación adecuados que se traduzcan en entusiasmo y amenidad en la entrega de enseñanzas. El trabajo grupal en nuestra disciplina siempre ha sido una metodología impor- tante para abordar simulaciones y casos, percibiéndolo así nuestros estudiantes, lo que debe ser un incentivo a preparar y aplicar estos en forma apropiada y guiada, tanto en el aula como fuera de ella.

La tecnología, si bien importante en la formación de los profesionales de hoy, su uso sólo será efectivo en el aula si esta se utiliza apropiadamente, no constituyéndose en muchas asignaturas en factor gravitante para los aprendizajes, así lo perciben los estudiantes.

Los estudiantes de Auditoría de la Universidad de Los Lagos ven en algunos de sus docentes las referencias profesionales a que aspiran tener, un honor y una gran responsabilidad a la vez, pues no sólo debemos esforzarnos por ser buenos docentes, cautelar adecuadamente los procesos de enseñanza - aprendizajes y la adquisición de competencias por parte de nuestros estudiantes, sino que nuestro deber traspasar el aula y la universidad, debiendo ser buenos profesionales y personas idóneas dentro de la sociedad.

\section{BIBLIOGRAFÍA}

A Polo, JM Hernández, C Pozo (1996). Evaluación del estrés académico en estudiantes universitarios. Scielo. Org. Full View.

Bernard, J. A. (1993). "Estrategias de aprendizaje y enseñanza: evaluación de una actividad compartida en la escuela", en C. MONEREO (Ed.):Las estrategias de aprendizaje. Procesos, contenidos e interacción. Barcelona, Domenech.

Bligh, D. A. (1971). What's the use of lecturing? Devon, England: Teaching Service Centre, University of Exeter.

Cano, F. y F. Justicia. (1998). Los estilos de aprendizaje en psicología y educación En Psicología de la instrucción: componentes cognitivos y afectivo de aprendizaje escolar. Barcelona: EUB.

Ericksen, S. C. (1978). The Lecture Memo to the Faculty, N60. Ann Arbor: Center for Research on teaching and learning, University of Michigan.

FD Betoret, PJ Jiménez, JR Remírez - Psicothema (2004). Percepción del proceso de enseñanza aprendizaje y su incidencia en el rendimiento.. - unioviedo. Scileo.org.pe 
Fedman, L Goncalves, G Chacon-Puignau (2008). Relaciones entre estrés académico y rendimiento en estudiantes. Universitas - scielo.org.co

García L., F. (2005). Motivar para el aprendizaje desde la actividad orientadora. La falta de motivación para el aprendizaje y su influencia en el rendimiento académico. España. Ministerio de Educación.

Genovard, C. y Gotzens, C. (1990). Psicología de la instrucción. Madrid: Santillana.

Melot, A.M. (1990). El conocimiento de los fenómenos psicológicos. En Monereo, C. (Ed.) Enseñar a pensar a través del currículo escolar. Barcelona: Casals.

P Bourdieu, JC Passeron (2009). Los herederos: Los estudiantes y la cultura. Obtenido de (http:/ / atlas.umss.edu.bo:8080/xmlui/handle/123456789/877). Consultado en abril de 2015.

Ramírez, J., Santander E. (2003). Instrumentos de evaluación a través de competencias. Santiago de Chile.

RM Méndez García (2009). Los estudiantes: su percepción sobre lo que implica "ser estudian- tes" y "hacer universidad". Revista de Investigación Educativa, 2009 - revistas.um.es.

Sass, E. J. (1989). "Motivation in the College Classroom: What students tell us. Teaching of Psychology, 16(2), 86-88.

Selltiz, C (1970). Métodos de investigación de las relaciones sociales. Madrid, España: Rialp.

Tobón, S. \& Rial, A. y otros (2006). Competencias Calidad y Educación Superior. Colombia: Colección Alma Mater

http://www.repositorioacademico.usmp.edu. pe/bitstream/usmp/628/3/maldonado r.pdf Consultado en abril de 2015.

http:/ / es.slideshare.net/ carlosierra/matriz-depercepciones-5076043 Consultado en abril de 2015.

https:// scholar.google.cl/scholar?q=tesis+ percepcion+de+los+estudiantes\&hl=es\&as $\mathrm{sdt}=0 \&$ as vis $=1 \&$ oi $=$ scholart\&sa $=X \& v e d=0$ CBk QgQMwAGoVChMIzr2C3NnVxwIVxoGQCh2i EAhm Consultado en abril de 2015.

http:/ / atlas.umss.edu.bo:8080/xmlui/handle/123456789/877 Consultado en abril de 2015 . 
ANEXO: ENCUESTA APLICADA

\begin{abstract}
MEDICIÓN DE PERCEPCIÓN DE LOS ESTUDIANTES DE AUDITORÍA RESPECTO A LAS TÉCNICAS DE ENSEÑANZA UTILIZADAS EN EL AULA POR SUS DOCENTES
\end{abstract}

\title{
Ante cada pregunta o planteamiento deberá indicar su grado de acuerdo en una escala de 1 a 7 , siendo 1 el menor grado y 7 el máximo
}

\begin{tabular}{|c|c|c|c|c|c|c|c|}
\hline & 1 & 2 & 3 & 4 & 5 & 6 & 7 \\
\hline \multicolumn{8}{|l|}{ Cualidades del docente } \\
\hline \multicolumn{8}{|l|}{$\begin{array}{l}\text { Es importante para mí el nivel de } \\
\text { entusiasmo que demuestra el docen- } \\
\text { te en el aula }\end{array}$} \\
\hline \multicolumn{8}{|l|}{$\begin{array}{l}\text { El dominio profesional en el área } \\
\text { (carrera) demostrado por el docente } \\
\text { me otorga mayor confianza }\end{array}$} \\
\hline \multicolumn{8}{|l|}{$\begin{array}{l}\text { El cumplimiento de fechas y hora- } \\
\text { rios por parte del docente es gravi- } \\
\text { tante }\end{array}$} \\
\hline \multicolumn{8}{|l|}{$\begin{array}{l}\text { El lenguaje del docente, tanto téc- } \\
\text { nico como general, incide en mis } \\
\text { aprendizajes }\end{array}$} \\
\hline \multicolumn{8}{|l|}{$\begin{array}{l}\text { La experiencia, así mismo el perfec- } \\
\text { cionamiento del docente son impor- } \\
\text { tantes para mi formación }\end{array}$} \\
\hline \multicolumn{8}{|l|}{$\begin{array}{l}\text { Prefiero docentes alegres, pero a la } \\
\text { vez justos en sus evaluaciones }\end{array}$} \\
\hline \multicolumn{8}{|l|}{$\begin{array}{l}\text { Los docentes deben tener grados } \\
\text { adecuados de independencia del } \\
\text { material de apoyo en el aula }\end{array}$} \\
\hline & 1 & 2 & 3 & 4 & 5 & 6 & 7 \\
\hline \multicolumn{8}{|l|}{ Material de apoyo } \\
\hline \multicolumn{8}{|l|}{$\begin{array}{l}\text { Los apuntes y guías de trabajo gene- } \\
\text { radas por los docentes constituyen } \\
\text { un apoyo importante a cada asig- } \\
\text { natura }\end{array}$} \\
\hline \multicolumn{8}{|l|}{$\begin{array}{l}\text { Valoro el material preparado o adap- } \\
\text { tado por los docentes para sus asig- } \\
\text { naturas por sobre el de otros autores }\end{array}$} \\
\hline \multicolumn{8}{|l|}{$\begin{array}{l}\text { Las lecturas y referencias bibliográ- } \\
\text { ficas entregadas por los docentes } \\
\text { deben ser analizadas en conjunto en } \\
\text { clases }\end{array}$} \\
\hline \multicolumn{8}{|l|}{$\begin{array}{l}\text { Los casos y su desarrollo en clases } \\
\text { ayudan en gran medida a mi proce- } \\
\text { so de aprendizaje }\end{array}$} \\
\hline $\begin{array}{l}\text { Es importante para mi tener orienta- } \\
\text { ción respecto a la referencia de ma- } \\
\text { terial de apoyo, tanto en biblioteca } \\
\text { como en la web }\end{array}$ & & & & & & & \\
\hline
\end{tabular}




\begin{tabular}{|c|c|c|c|c|c|c|c|}
\hline & 1 & 2 & 3 & 4 & 5 & 6 & 7 \\
\hline \multicolumn{8}{|l|}{ Organización } \\
\hline \multicolumn{8}{|l|}{$\begin{array}{l}\text { Es importante conocer la organiza- } \\
\text { ción de las asignaturas al inicio de } \\
\text { cada semestre }\end{array}$} \\
\hline \multicolumn{8}{|l|}{$\begin{array}{l}\text { La organización de la asignatura es } \\
\text { importante para mi }\end{array}$} \\
\hline \multicolumn{8}{|l|}{$\begin{array}{l}\text { Valoro en gran medida el cumpli- } \\
\text { miento de la planificación }\end{array}$} \\
\hline \multicolumn{8}{|l|}{$\begin{array}{l}\text { Las clases no efectuadas deben ser } \\
\text { recuperadas a través de algún meca- } \\
\text { nismo eficaz }\end{array}$} \\
\hline & 1 & 2 & 3 & 4 & 5 & 6 & 7 \\
\hline \multicolumn{8}{|l|}{ Nivel de dificultad } \\
\hline \multicolumn{8}{|l|}{$\begin{array}{l}\text { Prefiero las asignaturas "fáciles" por } \\
\text { sobre las más exigentes }\end{array}$} \\
\hline \multicolumn{8}{|l|}{$\begin{array}{l}\text { Valoro las asignaturas donde se } \\
\text { combina el nivel apropiado con téc- } \\
\text { nicas facilitadoras de aprendizajes }\end{array}$} \\
\hline \multicolumn{8}{|l|}{$\begin{array}{l}\text { Las asignaturas difíciles y / o com- } \\
\text { plejas no lo son tanto con buenos } \\
\text { docentes }\end{array}$} \\
\hline & 1 & 2 & 3 & 4 & 5 & 6 & 7 \\
\hline \multicolumn{8}{|l|}{ Participación activa } \\
\hline \multicolumn{8}{|l|}{$\begin{array}{l}\text { Valoro las clases interactivas donde } \\
\text { el docente hace participar a los es- } \\
\text { tudiantes }\end{array}$} \\
\hline \multicolumn{8}{|l|}{$\begin{array}{l}\text { Me agrada asumir un rol activo en } \\
\text { clases }\end{array}$} \\
\hline $\begin{array}{l}\text { Prefiero los docentes que fomentan } \\
\text { la participación activa en clases. }\end{array}$ & & & & & & & \\
\hline
\end{tabular}

\begin{tabular}{|c|c|c|c|c|c|c|c|}
\hline & 1 & 2 & 3 & 4 & 5 & 6 & 7 \\
\hline \multicolumn{8}{|l|}{ Uso de tecnología } \\
\hline \multicolumn{8}{|l|}{$\begin{array}{l}\text { Es importante el uso de tecnología } \\
\text { (Data Show, Computadores, etc.) por } \\
\text { parte de los docentes en el aula. }\end{array}$} \\
\hline \multicolumn{8}{|l|}{$\begin{array}{l}\text { El uso de tecnología por parte de los } \\
\text { docentes en el aula debe es siempre } \\
\text { ameno y didáctico }\end{array}$} \\
\hline $\begin{array}{l}\text { A veces prefiero una clase tradicio- } \\
\text { nal, sin uso de tecnología de apoyo. }\end{array}$ & & & & & & & \\
\hline
\end{tabular}




\begin{tabular}{|c|c|c|c|c|c|c|c|}
\hline & 1 & 2 & 3 & 4 & 5 & 6 & 7 \\
\hline \multicolumn{8}{|l|}{ Conexión profesor - estudiante } \\
\hline \multicolumn{8}{|l|}{$\begin{array}{l}\text { Adquiero aprendizajes y conoci- } \\
\text { mientos con mayor facilidad con los } \\
\text { docentes más empáticos. }\end{array}$} \\
\hline \multicolumn{8}{|l|}{$\begin{array}{l}\text { Me agrada que los docentes atien- } \\
\text { dan mis consultas e inquietudes } \\
\text { dentro y fuera del aula. }\end{array}$} \\
\hline $\begin{array}{l}\text { Visualizo a algunos docentes como } \\
\text { buenos referentes profesionales }\end{array}$ & & & & & & & \\
\hline
\end{tabular}

\begin{tabular}{|c|c|c|c|c|c|c|c|}
\hline & 1 & 2 & 3 & 4 & 5 & 6 & 7 \\
\hline \multicolumn{8}{|l|}{ Ejemplos - referencias } \\
\hline \multicolumn{8}{|l|}{$\begin{array}{l}\text { Me ayuda mucho que el docente } \\
\text { entregue ejemplos apropiados de } \\
\text { aplicación de contenidos }\end{array}$} \\
\hline \multicolumn{8}{|l|}{$\begin{array}{l}\text { Las referencias a situaciones profe- } \\
\text { sionales verídicas son un aporte a mi } \\
\text { formación }\end{array}$} \\
\hline $\begin{array}{l}\text { El análisis por parte del docente } \\
\text { de la contingencia nacional, tanto } \\
\text { administrativa como profesional, es } \\
\text { importante para mi formación }\end{array}$ & & & & & & & \\
\hline
\end{tabular}

\begin{tabular}{|c|c|c|c|c|c|c|c|}
\hline & 1 & 2 & 3 & 4 & 5 & 6 & 7 \\
\hline \multicolumn{8}{|l|}{$\begin{array}{l}\text { Técnicas de Evaluación (En activi- } \\
\text { dades disciplinares) }\end{array}$} \\
\hline \multicolumn{8}{|l|}{$\begin{array}{l}\text { Talleres y/o Evaluaciones grupales, } \\
\text { con asesoría del docente, posibilitan } \\
\text { mejor mis aprendizajes }\end{array}$} \\
\hline \multicolumn{8}{|l|}{$\begin{array}{l}\text { Prefiero por cuanto son más efecti- } \\
\text { vas, las evaluaciones individuales }\end{array}$} \\
\hline \multicolumn{8}{|l|}{$\begin{array}{l}\text { Considero un mix de trabajos de } \\
\text { investigación, talleres y evaluaciones } \\
\text { individuales más adecuado. }\end{array}$} \\
\hline $\begin{array}{l}\text { Las evaluaciones de proceso (parcia- } \\
\text { les) deben tener una incidencia por- } \\
\text { centual final gravitante (porcentaje } \\
\text { de la nota final) }\end{array}$ & & & & & & & \\
\hline
\end{tabular}

Me gustaría agregar.

\section{GRACIAS POR TU COLABORACIÓN}

